\title{
Procyanidin C1 from Cinnamomi Cortex inhibits TGF- $\beta$-induced epithelial-to-mesenchymal transition in the A549 lung cancer cell line
}

\author{
RYOEI KIN $^{1}$, SHINICHIRO KATO ${ }^{1}$, NAOKI KANETO ${ }^{1}$, HIROAKI SAKURAI ${ }^{1,2}$, YOSHIHIRO HAYAKAWA ${ }^{1}$, \\ FENG LI $^{2}$, KEN TANAKA ${ }^{3}$, IKUO SAIKI ${ }^{1}$ and SATORU YOKOYAMA ${ }^{1}$ \\ ${ }^{1}$ Division of Pathogenic Biochemistry, Institute of Natural Medicine, ${ }^{2}$ Department of Cancer Cell Biology, \\ Graduate School of Medicine and Pharmaceutical Science, ${ }^{3}$ Research Promotion Office, \\ ${ }^{4}$ Division of Pharmacognosy, Institute of Natural Medicine, University of Toyama, Toyama 930-0194, Japan
}

Received July 10, 2013; Accepted September 2, 2013

DOI: 10.3892/ijo.2013.2139

\begin{abstract}
Cancer metastasis is one of the most critical events in cancer patients, and the median overall survival of stage IIIb or IV patients with metastatic lung cancer in the TNM classification is only 8 or 5 months, respectively. We previously demonstrated that Juzentaihoto, a Japanese traditional medicine, can inhibit cancer metastasis through the activation of macrophages and $\mathrm{T}$ cells in mouse cancer metastatic models; however, the mechanism(s) through which Juzentaihoto directly affects tumor cells during the metastasis process and which herbal components from Juzentaihoto inhibit the metastatic potential have not been elucidated. In this study, we focused on the epithelial-to-mesenchymal transition (EMT), which plays an important role in the formation of cancer metastasis. We newly determined that only the Cinnamomi Cortex (CC) extract, one of 10 herbal components of Juzentaihoto, inhibits TGF- $\beta$-induced EMT. Moreover, the contents of catechin trimer in $\mathrm{CC}$ extracts were significantly correlated with the efficacy of inhibiting TGF- $\beta$-induced EMT. Finally, the structure of the catechin trimer from $\mathrm{CC}$ extract was chemically identified as procyanidin $\mathrm{C} 1$ and the compound showed inhibitory activity against TGF- $\beta$-induced EMT. This illustrates that procyanidin $\mathrm{C} 1$ is the main active compound in the $\mathrm{CC}$ extract responsible for EMT inhibition and that procyanidin $\mathrm{C} 1$ could be useful as a lead compound to develop inhibitors of cancer metastasis and other diseases related to EMT.
\end{abstract}

Correspondence to: Dr Satoru Yokoyama, Institute of Natural Medicine, University of Toyama, 2630 Sugitani, Toyama 930-0194, Japan

E-mail: yokoyama@inm.u-toyama.ac.jp

Abbreviations: EMT, epithelial-to-mesenchymal transition; CC, Cinnamomi Cortex

Key words: Cinnamomi Cortex, epithelial-to-mesenchymal transition, lung cancer, procyanidin $\mathrm{C} 1$

\section{Introduction}

The essential characteristics of cancer are the ability to invade surrounding tissues and metastasize to distal tissues, known as cancer metastasis, which is the major cause of mortality in cancer patients. In non-small cell lung cancer (NSCLC) patients, the median overall survival of patients with metastatic lung cancer (stage IIIb or IV in the TNM classification) is limited (only 8 or 5 months, respectively) (1); therefore, suppression of cancer metastasis results in the improved survival of lung cancer patients.

Cancer metastasis consists of several steps: intravasation, attachment to a vessel, extravasation, angiogenesis and growth in distal tissues (2-5). Among them, epithelial-to-mesenchymal transition (EMT) is involved in an early step of metastasis $(6,7)$. EMT is a phenomenon in which cobblestone-like epithelial cells change into spindle-like mesenchymal cells with downregulation of epithelial markers such as E-cadherin and also upregulation of mesenchymal markers such as N-cadherin (8). In addition to metastasis, this physiological phenomenon is important for resistance to apoptosis, maintenance of cancer stem cells and production of extracellular matrix. Thus, EMT would be an attractive therapeutic target in metastatic cancers.

Juzentaihoto, which is a Japanese Kampo medicine, has been widely used for the decline of physical strength, general debility, cold hands and feet, fatigue, night sweats, circulatory problems and anemia (9). Interestingly, our previous studies showed that Juzentaihoto indirectly inhibited cancer metastasis through the activation of macrophages and $\mathrm{T}$ cells in mouse models (10-12). Although Juzentaihoto could suppress cancer metastasis, it is not well known how Juzentaihoto can directly affect tumor cells during the metastasis process and which herbal ingredients of Juzentaihoto are involved in the regulation of EMT.

Here we firstly report that Cinnamomi Cortex (CC), one of the herbal medicines from Juzentaihoto, inhibits TGF- $\beta$ induced EMT phenotypes. After fractionation of CC extract, the content of catechin trimer was well-associated with the inhibitory activities of CC extract. Finally, procyanidin $\mathrm{C} 1$ 
from CC extract is newly identified as the responsible molecule for the EMT inhibition.

\section{Materials and methods}

Herbalmedicine extraction.Astagali Radix (AsR), Glycyrrhizae Radix (GIR), Cinnamomi Cortex (CC), Rehmanniae Radix (RR), Paeoniae Radix (PR), Cnidii Rhizoma (CR), Angelicae Radix (AnR), Ginseng Radix (GiR), Hoelen (Ho), and Atractylodis Lanceae Radix (ALR) were purchased from Uchida-Wakanyaku Co., Ltd. (Tokyo, Japan). In this study, Cinnamomi Cortex was added to an appropriate volume of distilled water $(\mathrm{w} / \mathrm{v}, 1: 10)$ and extracted at $100^{\circ} \mathrm{C}$ for $1 \mathrm{~h}$. The extracted solution was filtered and then freeze dried to obtain dried powder. A voucher sample of this extract (INM 10000007, University of Toyama) was preserved in the Research Promotion Office, Institute of Natural Medicine, University of Toyama, Toyama, Japan. Six other Cinnamomi Cortex samples were collected from different regions of Vietnam and China, coded as CC-1 (Vietnam), CC-2 (Guang Xi, China), CC-3 (Guang Xi, China), CC-4 (Guang Dong, China), CC-5 (Guang Xi, China), CC-6 (Vietnam). Water extracts of the above-mentioned six CC samples were prepared and provided by the National Institute of Biomedical Innovation, Osaka, Japan, and a part of each extract was deposited at our institute (voucher specimen no. INM 10000001-10000006).

Cell cultures. Human lung adenocarcinoma A549 cells were cultured in RPMI-1640 medium (Invitrogen, Carlsbad, CA, USA) with $10 \%$ fetal bovine serum (FBS; ICN Biomedicals, Aurora, OH, USA), 2 mM L-glutamine (Invitrogen), $100 \mathrm{U} / \mathrm{ml}$ penicillin and $100 \mu \mathrm{g} / \mathrm{ml}$ streptomycin in $5 \% \mathrm{CO}_{2}$ at $37^{\circ} \mathrm{C}$. The cells were treated with recombinant human TGF- $\beta(5 \mathrm{ng} / \mathrm{ml})$ (Peprotech, London, UK) for various times as indicated, after pretreatment with TGF- $\beta$ receptor I kinase inhibitor $(10 \mu \mathrm{M})$ (Merck, Whitehouse, NJ, USA), each herbal component from Juzentaihoto, or Cinnamomi Cortex extracts (CC, or CC-1 to CC-6) for $30 \mathrm{~min}$.

Protein preparation and western blotting. Whole cell lysates were collected in lysis buffer supplemented with some protease and phosphatase inhibitors as described previously (13). Equal amounts of protein were resolved by electrophoresis on acrylamide gels and transferred to PVDF membranes. Antibodies against phospho-specific Smad-2 (Ser 465/467), E-cadherin, and $\mathrm{N}$-cadherin were purchased from Cell Signaling Technology (Beverly, MA, USA) and an antibody against PCNA was purchased from Santa Cruz Biotechnology (Santa Cruz, CA, USA).

Cell morphology and cell migration. Cell morphology was determined by BZ-8000 (Keyence, Osaka, Japan) after staining with hematoxylin and eosin. For the migration assay, Transwell cell culture chambers were used as described previously (14). Briefly, the filters were precoated with $1.25 \mu \mathrm{g}$ fibronectin on the lower surfaces. The cell suspension $\left(3 \times 10^{4}\right.$ cells $\left./ 100 \mu \mathrm{l}\right)$ in serum-free medium was added to the upper compartment and incubated for $6 \mathrm{~h}$. The migrated cells were stained with hematoxylin and eosin, and counted under the microscope in three predetermined fields at a magnification of $\mathrm{x} 400$.
Fractionation of $C C$ and identification of procyanidin $C 1$ from $C C$. CC extract, $2.0 \mathrm{~g}$, was subjected to reversedphase silica gel (Cosmosil $75 \mathrm{C}_{18}-\mathrm{OPN}$; Nacalai Tesque Inc., Kyoto, Japan) using medium pressure liquid chromatography (MPLC; Buchi, Flawil, Switzerland) with a $\mathrm{H}_{2} \mathrm{O}-\mathrm{CH}_{3} \mathrm{CN}$ gradient system $(98: 2 \rightarrow 96: 4 \rightarrow 92: 8 \rightarrow 90: 10 \rightarrow 80: 20 \rightarrow 60: 40 \rightarrow 40: 60$ $\rightarrow 10: 90$ ) to obtain eight fractions (fr. 1, $435 \mathrm{mg}$; fr. 2, $282 \mathrm{mg}$; fr. 3, $123 \mathrm{mg}$; fr. 4, $212 \mathrm{mg}$; fr. 5, $105 \mathrm{mg}$; fr. 6, $305 \mathrm{mg}$; fr. 7, $205 \mathrm{mg}$; fr. 8, $105 \mathrm{mg}$ ). The bioactive fraction 4 (fr. 4, $200 \mathrm{mg}$ ) was further subjected to preparative HPLC (Discovery C18 column; 10x250 mm i.d., $5 \mu \mathrm{m}$ particle size; Supelco, PA, USA) with $\mathrm{H}_{2} \mathrm{O}-\mathrm{CH}_{3} \mathrm{CN}(92: 8)$ containing $0.01 \%$ trifluoroacetic acid (TFA) at a flow rate of $2 \mathrm{ml} / \mathrm{min}$ to yield a procyanidin trimer $\left(\mathbf{1}, 5.5 \mathrm{mg}, t_{\mathrm{R}} 23.3 \mathrm{~min}\right)$. The molecular formula of compound 1 was determined by HR-TOF-MS to be $\mathrm{C}_{45} \mathrm{H}_{38} \mathrm{O}_{18}[\mathrm{~m} / \mathrm{z} 865.2003$ $(\mathrm{M}-\mathrm{H})^{+}$]. Its chemical structure was further identified to be epicatechin- $(4 \beta \rightarrow 8)$-epicatechin- $(4 \beta \rightarrow 8)$-epicatechin (procyanidin $\mathrm{C} 1$, Fig. $3 \mathrm{C}$ ) by comparing the $\left[{ }^{1} \mathrm{H}\right]$ nuclear magnetic resonance (NMR), $\left[{ }^{13} \mathrm{C}\right] \mathrm{NMR}$, and circular dichroism (CD) spectral data with those in the literature (15).

Liquid chromatography-mass spectrometry (LC-MS) analysis. For chemical profiling of $7 \mathrm{CC}$ extracts, liquid chromatography-mass spectrometry (LC-MS) analysis was performed with a Shimadzu LC-IT-TOF mass spectrometer (Kyoto, Japan) equipped with an ESI interface (Shimadzu). The ESI parameters were as follows: source voltage $+4.5 \mathrm{kV}$, capillary temperature $200^{\circ} \mathrm{C}$ and nebulizer gas $1.5 \mathrm{l} / \mathrm{min}$. The mass spectrometer was operated in positive ion mode scanning from $\mathrm{m} / \mathrm{z}, 200$ to 2,000. A Waters Atlantis T3 column (2.1 mm i.d. x $150 \mathrm{~mm}, 3 \mathrm{~m}$; Milford, MA, USA) was used and the column temperature was maintained at $40^{\circ} \mathrm{C}$. The mobile phase was a binary eluent of (A) $5 \mathrm{mM}$ ammonium acetate solution and (B) $\mathrm{CH}_{3} \mathrm{CN}$ under the following gradient conditions: 0-30 min linear gradient from 10 to $100 \% \mathrm{~B}, 30-40 \mathrm{~min}$ isocratic at $100 \% \mathrm{~B}$. The flow rate was $0.15 \mathrm{ml} / \mathrm{min}$. Mass spectrometry data obtained from the extract were deposited in the MassBank Database and stored with pharmacological information on the extract in the Wakan-Yaku Database System, Institute of Natural Medicine, University of Toyama.

\section{Results}

Cinnamomi Cortex (CC) extract suppresses TGF- $\beta$-induced EMT. Our previous studies showed that Juzentaihoto, a Japanese Kampo medicine, prevents metastasis in mouse models (10-12). Because Juzentaihoto contains ten ('Ju' means 'ten' in Japanese) kinds of herbal ingredients, we thus investigated whether each component can suppress TGF- $\beta$-induced EMT. We firstly screened the expression of an epithelial marker, E-cadherin, in a human non-small-cell lung cancer cell line, A549 cells, treated with TGF- $\beta$, TGF- $\beta$ receptor kinase inhibitor (TGFRi), or herbal medicines (Fig. 1A). Known as EMT, the reduction of E-cadherin expression in A549 cells was detected after TGF- $\beta$ treatment in A549 cells. Among the ten herbal components, only Cinnamomi Cortex (CC) extract strikingly suppressed TGF- $\beta$-induced EMT, as indicated by the restoration of E-cadherin. We could not detect any inhibition of the cell viabilities by CC extract (data not shown). 


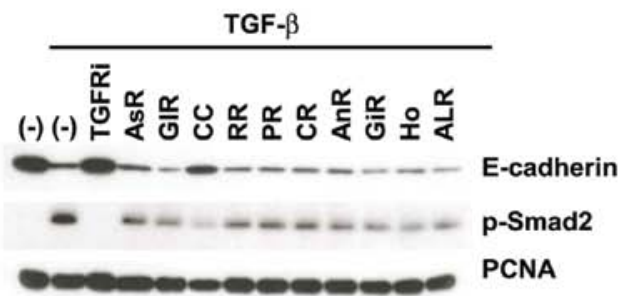

B

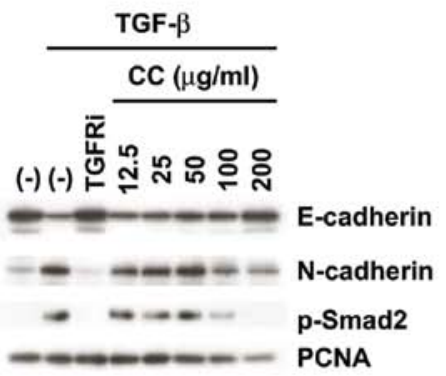

C

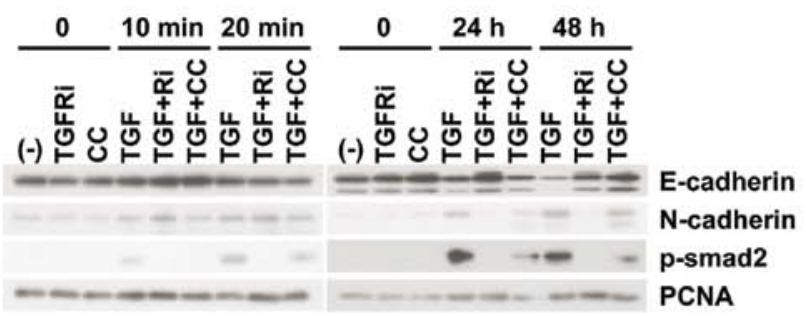

D

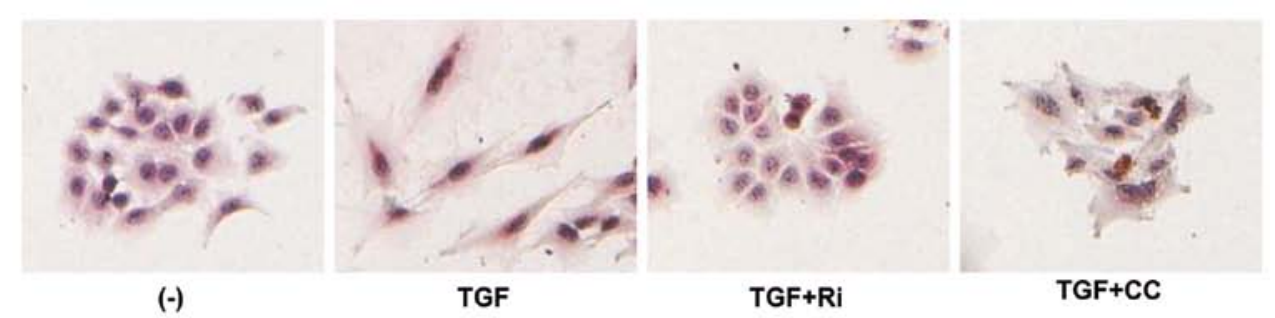

E

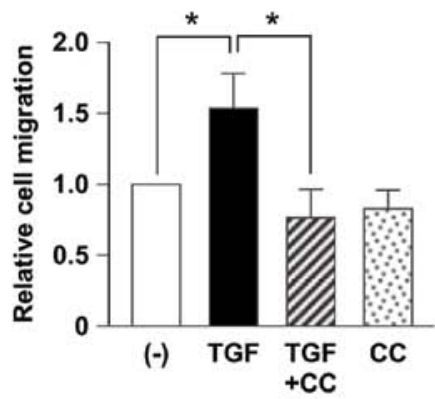

Figure 1. Cinnamomi Cortex (CC) suppresses TGF- $\beta$-induced EMT. (A) Effects of ten herbal components from Juzentaihoto. AsR, Astagali Radix; GlR, Glycyrrhizae Radix; CC, Cinnamomi Cortex; RR, Rehmanniae Radix; PR, Paeoniae Radix; CR, Cnidii Rhizoma; AnR, Angelicae Radix, GiR, Ginseng Radix; Ho, Hoelen; ALR, Atractylodis Lanceae Radix. A549 cells were pretreated with various herbal ingredients (100 $\mu \mathrm{g} / \mathrm{ml})$ or TGF- $\beta$ receptor I kinase inhibitor (TGFRi; $10 \mu \mathrm{M}$ ) for $30 \mathrm{~min}$ and stimulated with TGF- $\beta(5 \mathrm{ng} / \mathrm{ml}$ ) for $48 \mathrm{~h}$. Expression of each protein was detected by western blotting. (B) Dose-dependent inhibition of TGF- $\beta$-induced EMT with CC treatment. A549 cells were pretreated with various concentrations of CC $(12.5,25,50,100 \mathrm{and} 200 \mu \mathrm{g} / \mathrm{ml})$ or TGF- $\beta$ receptor I kinase inhibitor (TGFRi; $10 \mu \mathrm{M}$ ) for $30 \mathrm{~min}$ and stimulated with TGF- $\beta(5 \mathrm{ng} / \mathrm{ml}$ ) for $48 \mathrm{~h}$. Expression of each protein was detected by western blotting. (C) Time-dependent inhibition of TGF- $\beta$-induced EMT with CC treatment. A549 cells were pretreated with CC (100 $\mu \mathrm{g} / \mathrm{ml})$ for $30 \mathrm{~min}$ and stimulated with TGF- $\beta(5 \mathrm{ng} / \mathrm{ml})$ for the indicated times. Expression of each protein was detected by western blotting. (D) Cell morphologies with CC treatment. A549 cells were stained by H\&E after treatment as in (C) for $48 \mathrm{~h}$. (E) Migration with CC treatment. A549 cells treated as in (D) were seeded in Transwell chambers for $6 \mathrm{~h}$. No. of migrated cells per field (x400) was counted. Relative cell migration with TGF- $\beta$ (TGF, filled bar), TGF- $\beta$ with CC extract (TGF+CC, shadow bar), or $\mathrm{CC}$ extract (CC, dotted bar) was normalized by untreated cell migration (open bar). Data are the mean \pm SD of four independent experiments. ${ }^{*}<0.01$, compared among each pair, by one-way ANOVA and Bonferroni's multiple comparision test. 
A
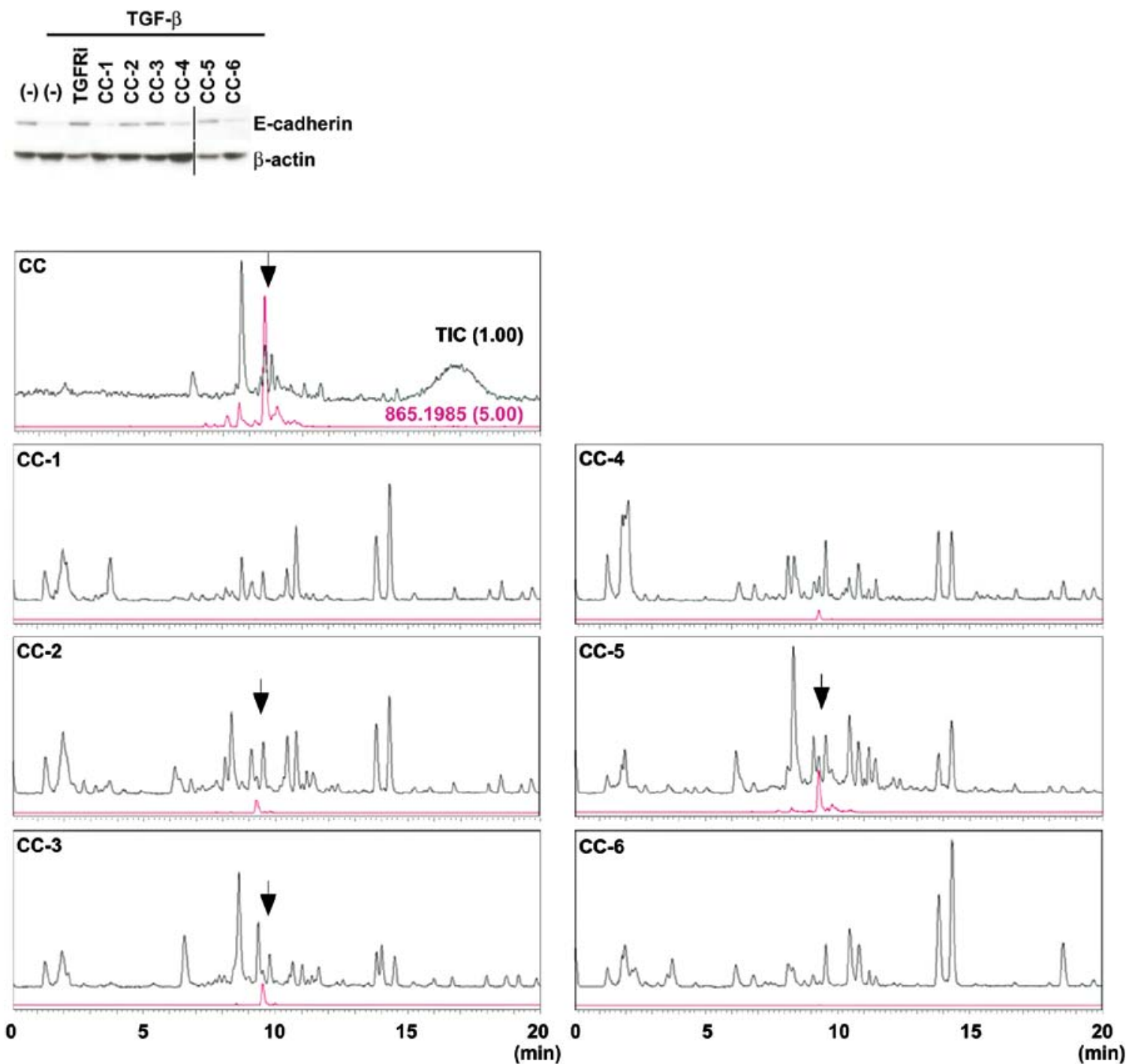

Figure 2. CCs show variability in the activity of TGF- $\beta$-induced EMT. (A) Effects of various CCs. A549 cells were pretreated with various CCs (100 $\mu \mathrm{g} / \mathrm{ml}$ ), but other conditions were similar to Fig. 1C. Expression of each protein was detected by western blotting. (B) Inhibition activities of CCs correlated with the content of catechin trimer. Total ion chromatogram (TIC) profiles of various CC extracts are shown. Arrows show the catechin trimer fractions.

In order to further determine the inhibition of TGF- $\beta$ induced EMT by CC extract, E-cadherin, $\mathrm{N}$-cadherin, and phosphorylated Smad-2 were examined. Similarly to Fig. 1A, restoration of E-cadherin expression by $\mathrm{CC}$ extract was detected in a concentration/time-dependent manner (Fig. 1B and $\mathrm{C}$ ). Interestingly, phosphorylation of Smad-2 by TGF- $\beta$, a downstream molecule of TGF- $\beta$ signaling, was suppressed by $\mathrm{CC}$ extract after the early phase $(10$ and $20 \mathrm{~min})$ as well as the late phase (24 and $48 \mathrm{~h})$. We also detected the inhibition of TGF- $\beta$-induced EMT by CC extract at mRNA levels of snail, E-cadherin and fibronectin (data not shown). In addition to expression levels of EMT markers, we observed morphological changes with CC extract (Fig. 1D). A549 cells treated with TGF- $\beta$ showed spindle-like shapes as compared with untreated cells. On the other hand, TGF- $\beta$-stimulated cells treated together with $\mathrm{CC}$ extract showed cobblestone-like shapes similar to the untreated cells or the cells with TGFRi and TGF- $\beta$. Moreover, consistent with cell morphology, TGF- $\beta$ - induced cell migration was also suppressed with $\mathrm{CC}$ extract (Fig. 1E). These results support that CC extract suppresses TGF- $\beta$-induced EMT in A549 cells and consequently inhibits cell migration.

Identification of an active fraction inhibiting TGF- $\beta$-induced $E M T$. To gain insight into the variations of $\mathrm{CC}$ extracts in EMT-inhibitory activities, we examined six additional CC (CC-1 to -6) extracts, which differ according to the harvest location (Fig. 2A). CC-2, CC-3 and CC-5 extracts showed restoration of E-cadherin expression stronger than $\mathrm{CC}-1$, CC-4, and CC- 6 extracts; therefore, an active component(s) for the inhibition of EMT might be included in CC-2, CC-3 and CC-5 extracts more than in CC-1, CC-4 and CC-6.

To identify the active chemical compound(s) that is able to inhibit TGF- $\beta$-induced EMT, total ion chromatogram analysis was performed in CC and CC- 1 to CC- 6 extracts. Notably, the peaks of catechin-catechin-catechin trimer (catechin trimer) 


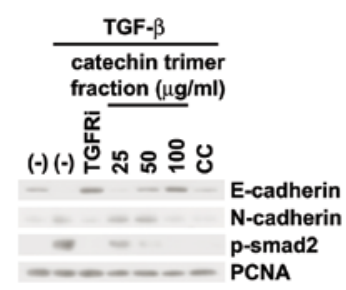

B
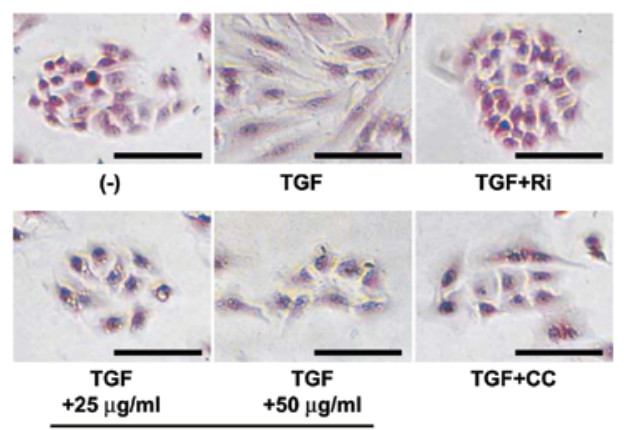

catechin trimer fraction

C

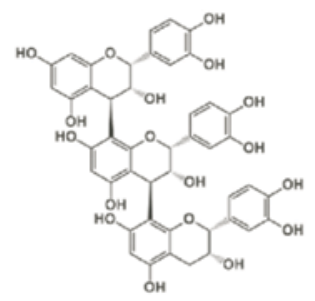

D

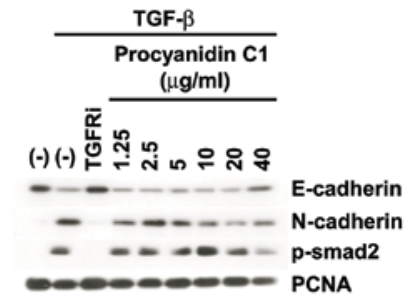

E

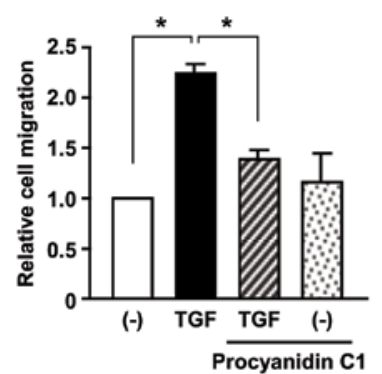

Figure 3. Procyanidin C1 from CC extract inhibits TGF- $\beta$-induced EMT. (A) Effects of catechin trimer fraction. A549 cells were pretreated with catechin trimer at the indicated concentration for $30 \mathrm{~min}$, but other conditions are similar to Fig. 1C. Expression of each protein was detected by western blotting. (B) Cell morphologies with catechin trimer fraction. A549 cells were stained by $\mathrm{H} \& \mathrm{E}$ after treatment as in Fig. $1 \mathrm{C}$ for $48 \mathrm{~h}$. (C) Structure of procyanidin C1. (D) Dose-dependent inhibition of TGF- $\beta$-induced EMT with procyanidin $\mathrm{C} 1$ from $\mathrm{CC}$. Other conditions were similar to (A). Expression of each protein was detected by western blotting. (E) Migration with procyanidin $\mathrm{C} 1$ from CC. A549 cells were treated as in (C). Relative cell migration of the cells treated with TGF- $\beta$ (TGF, filled bar), with both TGF- $\beta$ and procyanidin $\mathrm{C} 1$ (shadow bar) or with procyanidin $\mathrm{C} 1$ (dotted bar) was normalized by untreated cells (open bar). Data are the mean \pm SD of three independent experiments. " $\mathrm{p}<0.01$, compared among each pair, by one-way ANOVA and Bonferroni's multiple comparision test. significantly overlapped with EMT inhibitory activities in CC-2, CC-3 and CC-5 extracts, but were not detected in $\mathrm{CC}-1$, CC-4, and CC-6 extracts (Fig. 2B), suggesting that the catechin trimer may have crucial roles in suppressing TGF- $\beta$-induced EMT.

Procyanidin C1 can suppress TGF- $\beta$-induced EMT. Next to assess whether catechin trimer fraction of $\mathrm{CC}$ extract has activity to inhibit EMT, we examined the protein expression levels with the catechin trimer fraction from CC (Fig. 3A). As predicted, the catechin trimer fraction inhibited E-cadherin reduction and $\mathrm{N}$-cadherin induction. Similarly to protein levels, the change of cell morphology (Fig. 3B) also supported the inhibition of TGF- $\beta$-induced EMT.

We finally purified the catechin trimer from $\mathrm{CC}$ and identified its structure, which was an epicatechin-epicatechinepicatechin trimer, procyanidin $\mathrm{C} 1$ (Fig. 3C). To check the inhibition of EMT by procyanidin $\mathrm{C} 1$, the protein expression and cell migration activities were examined (Fig. 3D and E). Strikingly, EMT markers represented the inhibition of EMT in western blotting, and TGF- $\beta$-induced cell migration was suppressed by procyanidin $\mathrm{C} 1$.

\section{Discussion}

In this study, we firstly identified Cinnamomi Cortex (CC) extract in 10 herbal components from Juzentaihoto as the only inhibitory herbal ingredient of TGF- $\beta$-induced epithelial-tomesenchymal transition (EMT) in a human non-small cell lung cancer cell line, A549 cells. The inhibitory effects of CC extracts could be derived from the procyanidin $\mathrm{C} 1$ because of the correlation of the content of procyanidin $\mathrm{C} 1$ in $\mathrm{CC}$ extract with their inhibitory activities.

The inhibitory effect of CC extract on EMT phenotypes we showed here might be supported by the evidence gained from Ninjinyoeito and Keishibukuryogan, both of which are other Kampo medicines containing CC. Ninjinyoeito has also been reported to suppress cancer metastasis (16). On the other hand, Keishibukuryogan has been shown to suppress renal fibrosis, which is caused by TGF- $\beta$-induced EMT (17). Despite the inhibitory activity of $\mathrm{CC}$ extract, the possibility can not be excluded that other herbal medicines might play a role in concert with $\mathrm{CC}$ extract, because of the harmonization effects among various herbal medicines $(11,12)$.

We identified procyanidin $\mathrm{C} 1$ as an active compound for the inhibition of EMT, but CC extract contains various chemical compounds, such as phenyl propanoids (cinnamic acid, cinnamaldehyde), terpenoids (cinnamonol, cassioside) and tannin (epicatechin) (18). There are many reports on the biological activities of cinnamic acid or cinnamaldehyde, for example, induction of ROS-mediated apoptosis (19). In addition, the procyanidin $\mathrm{C} 1$ we purified is known for its anti-inflammatory effects $(20,21)$; however, it is the first time to show that procyanidin $\mathrm{C} 1$ has anti-metastatic or anti-EMT effects. Among the catechin sub-family, epigallocatechin-3 gallate in green tea has been reported to inhibit EMT in human melanoma cells $(22,23)$. These reports raise the possibility that another catechin sub-family is involved in the inhibition of EMT besides procyanidin $\mathrm{C} 1$. The inhibition of cell migration by procyanidin $\mathrm{C} 1$ (Fig. 3E) and the clear linkage 
of the amount of procyanidin $\mathrm{C} 1$ to the inhibition of TGF- $\beta$ induced EMT (Fig. 2B) suggest that procyanidin $\mathrm{C} 1$ is still the main compound in $\mathrm{CC}$ extracts to inhibit cell migration and EMT.

Although we need more efforts to identify the target molecule of procyanidin $\mathrm{C} 1$, the phosphorylation of Smad-2 (Fig. 3D) and its transcriptional activity (data not shown) were suppressed. Indeed, $\mathrm{CC}$ extract and procyanidin $\mathrm{C} 1 \mathrm{did}$ not affect basal cell migration in A549 cells (CC alone or procyanidin $\mathrm{C} 1$ alone in Figs. 1E and $3 \mathrm{E}$ ), suggesting that the inhibition of EMT by $\mathrm{CC}$ extract and procyanidin $\mathrm{Cl}$ is dependent on inhibition of the TGF- $\beta$ signal pathway and that mesenchymal-to-epithelial transition is not induced by $\mathrm{CC}$ extract or procyanidin $\mathrm{C} 1$, which might affect basal levels of cell migration.

Collectively, our data identified CC in Juzentaihoto as the only herbal component inhibiting TGF- $\beta$-induced EMT, which could be worthy of clinical study in a variety of settings associated with EMT, including cancer metastasis and/or tissue fibrosis. As procyanidin $\mathrm{C} 1$ showed inhibition of EMT, it is suggested that the utilization of procyanidin $\mathrm{C} 1$ as a lead compound would be attractive for the development of cancer metastasis inhibitors.

\section{Acknowledgements}

This study was supported in part by Grants-in-Aid for Challenging Exploratory Research 24659348 (I.S.), by Grantin-Aid 24700971 for Young Scientists (B) (S.Y.) from the Ministry of Education, Culture, Sports, Science, and Technology (Japan) and by a Grant-in-Aid for the Cooperative Research Project from Joint Usage/Research Center (Joint Usage/ Research Center for Science-Based Natural Medicine), Institute of Natural Medicine, University of Toyama in 2013.

\section{References}

1. Ou SH and Zell JA: Validation study of the proposed IASLC staging revisions of the T4 and $M$ non-small cell lung cancer descriptors using data from 23,583 patients in the California Cancer Registry. J Thorac Oncol 3: 216-227, 2008.

2. Fidler IJ: The evolution of biological heterogeneity in metastatic neoplasms. In: Cancer Invasion and Metastases: Biologic and Therapeutic Aspects. Nicolson GL and Milas L (eds). Raven Press, New York, NY, pp5-26, 1984.

3. Nicolson GL: Tumor cell instability, diversification, and progression to the metastatic phenotype from oncogene to oncofetal expression. Cancer Res 47: 1473-1487, 1987.

4. Hart IR: Seed and soil revisited: mechanisms of site specific metastasis. Cancer Metastasis Rev 1: 5-16, 1982.
5. Liotta LA, Rao CV and Barsky SH: Tumour invasion and the extracellular matrix. Lab Invest 49: 636-649, 1983.

6. Kalluri R and Weinberg RA: The basics of epithelial-mesenchymal transition. J Clin Invest 119: 1420-1428, 2009.

7. Guarino M: Epithelial-mesenchymal transition and tumor invasion. Int J Biochem Cell Biol 39: 2153-2160, 2007.

8. Xu J, Lamouille S and Derynck R: TGF- $\beta$-induced epithelial to mesenchymal transition. Cell Res 19: 156-172, 2009.

9. Maruyama H, Kawamura H, Takemoto N, Komatsu Y, Aburaya $\mathrm{M}$ and Hosoya E: Effect of Juzentaihoto on phagocytes. Jpn J Inflammation 8: 461-465, 1988.

10. Ohnishi Y, Fujii H, Hayakawa Y, et al: Oral administration of a Kampo (Japanese herbal) medicine Juzentaihoto inhibits liver metastasis of colon 26-L5 carcinoma cells. Jpn J Cancer Res 89: 206-213, 1998

11. Ohnishi Y, Yamaura T, Tauchi K, et al: Expression of the antimetastatic effect induced by Juzentaihoto is based on the content of Shimotsu-to constituents. Biol Pharm Bull 7: 761-765, 1998.

12. Saiki I: A Kampo medicine 'Juzentaihoto' - prevention of malignant progression and metastasis of tumor cells and the mechanism of action. Biol Pharm Bull 23: 677-688, 2000.

13. Sakurai H, Suzuki S, Kawasaki N, et al: Tumor necrosis factoralpha-induced IKK phosphorylation of NF-kappaB p65 on serine 536 is mediated through the TRAF2, TRAF5, and TAK1 signaling pathway. J Biol Chem 278: 36916-36923, 2003.

14. Ogasawara M, Murata J, Ayukawa K and Saiki I: Differential effect of intestinal neuropeptides on invasion and migration of colon carcinoma cells in vitro. Cancer Lett 119: 125-130, 1997.

15. Esatbeyoglu T, Jaschok-Kentner B, Wray V and Winterhalter P: Structure elucidation of procyanidin oligomers by lowtemperature ${ }^{1} \mathrm{H}$ NMR spectroscopy. J Agric Food Chem 59: 62-69, 2011.

16. Kamei T, Kumano H, Iwata K, Nariai Y and Matsumoto T: The effect of a traditional Chinese prescription for a case of lung carcinoma. J Altern Complement Med 6: 557-579, 2000.

17. Nakagawa T, Tashiro I, Fujimoto M, et al: Keishibukuryogan reduces renal injury in the early stage of renal failure in the remnant kidney model. Evid Based Complement Alternat Med 2011: 914249, 2011.

18. Leela NK: 7 Cinnamon and Cassia. In: Chemistry of Spices. Parthasarathy VA, Chempakam B and Zachariah TJ (ed). CAB International, Wallingford, Oxon, pp124-145, 2008.

19. Ka H, Park HJ, Jung HJ, et al: Cinnamaldehyde induces apoptosis by ROS-mediated mitochondrial permeability transition in human promyelocytic leukemia HL-60 cells. Cancer Lett 196: $143-152,2003$.

20. Nakano N, Nishiyama C, Tokura T, et al: Procyanidin C1 from apple extract inhibits FceRI-mediated mast cell activation. Int Arch Allergy Immunol 147: 213-221, 2008.

21. Terra X, Palozza P, Fernandez-Larrea J, et al: Procyanidin dimer $\mathrm{B} 1$ and trimer $\mathrm{C} 1$ impair inflammatory response signalling in human monocytes. Free Radic Res 45: 611-619, 2011.

22. Singh T and Katiyar SK: Green tea catechins reduce invasive potential of human melanoma cells by targeting COX-2, PGE2 receptors and epithelial-to-mesenchymal transition. PLoS One 6: e25224, 2011.

23. Chen PN, Chu SC, Kuo WH, Chou MY, Lin JK and Hsieh YS: Epigallocatechin-3 gallate inhibits invasion, epithelial-mesenchymal transition, and tumor growth in oral cancer cells. J Agric Food Chem 59: 3836-3844, 2011. 